\title{
Decoupled Location Parameter Estimation of 3-D Near-Field Sources in a Uniform Circular Array using the Rank Reduction Algorithm
}

\author{
Tae-Jin Jung*, Bum-Soo Kwon*, and Kyun Kyung Lee* \\ *Kyungpook National University, Korea \\ (Received January 12, 2011; accepted March 16, 2011)
}

\begin{abstract}
An algorithm is presented for estimating the 3-D location (i.e., azimuth angle, elevation angle, and range) of multiple sources with a uniform circular array (UCA) consisting of an even number of sensors. Recently the rank reduction (RARE) algorithm for partly-calibrated sensor arrays was developed. This algorithm is applicable to sensor arrays consisting of several identically oriented and calibrated linear subarrays. Assuming that a UCA consists of M sensors, it can be divided into $\mathrm{M} / 2$ identical linear subarrays composed of two facing sensors. Based on the structure of the subarrays, the steering vectors are decomposed into two parts: range-independent 2-D direction-of-arrival (DOA) parameters, and range-relevant 3-D location parameters. Using this property we can estimate range-independent 2-D DOAs by using the RARE algorithm. Once the 2-D DOAs are available, range estimation can be obtained for each source by defining the 1-D MUSIC spectrum. Despite its low computational complexity, the proposed algorithm can provide an estimation performance almost comparable to that of the 3-D MUSIC benchmark estimator.
\end{abstract}

Keywords: Near-Field, Rank Reduction, UCA, Decoupled 3-D Source Localization, 3-D MUSIC

Subject classification: Underwater Transducers and Sonar System (5.6)

\section{Introduction}

Source localization using arrays of sensors is an important topic in wireless communications, radar, and sonar research [1-7]. One popular method is to employ a uniform circular array (UCA). From the various arrays available, a UCA offers an attractive geometry because it provides 2-D direction-of-arrival (DOA), 360 -azimuth coverage, and an almost identical beam width. By contrast, a uniform linear array (ULA) only gives a $1-D$ DOA (e.g. a bearing angle),

Corresponding author: Kyun Kyung Lee (kklee@ee.knu.ac.kr) IT-1 827, School of Electronics Engineering, College of IT Engineering, Kyungpook National University, 1370, Sankyukdong, Buk-gu, Daegu, 702-701, KOREA $180^{\circ}$ coverage, and a broadened beam width steered away from its broadside. Early research on source localization with a UCA focused primarily on far-field source localization, where source location was characterized by the 2-D DOA of the signal because the wavefront from a farfield source was assumed to be planar [1-2]. However, this assumption is no longer valid when the source is close to the UCA, in which case the location must be characterized by the range for the source as well as the 2-D DOA. Recently, some near-field 3-D source localization algorithms have been proposed using a UCA. Lee et al. [3] proposed a path-following algorithm based on the algebraic relation between the incident angles under the far-field assumption 
and the actual near-field location, which allows a 3-D location search to be replaced with a 2-D search for a far-field 2-D DOA estimation and a $1-D$ search following the known algebraic path. Bae et al. [4] developed a decoupled estimator for the 2-D DOA and range based on the centrosymmetry of the UCA and the noncircularity of the source, which allows a computationally efficient search-free near-field 3-D location estimator.

In addition, Pesavento et al. [5] developed a rank reduction estimator (RARE) algorithm that is a generalization of the multiple signal classification (MUSIC) algorithm [6] for partlycalibrated sensor arrays. This algorithm is applicable to sensor arrays consisting of several identically oriented and calibrated linear subarrays with the inter-element spacing of each subarray being an integer multiple of some common baseline. therefore this paper represents a decoupled location parameter estimator of multiple 3-D near-field sources in a UCA. A UCA is composed of $M / 2$ identical linear subarrays consisting of two facing sensors each. Based on the structure of the subarrays, the steering vectors can be decomposed into range-independent 2-D DOAs matrices and range-dependent $3-\mathrm{D}$ location parameter vectors. Hence, we can estimate the rangeindependent 2-D DOAs by using the RARE algorithm. Once the 2-D DOAs are available, the range estimation for each source can be obtained by defining the 1-D MUSIC spectrum. As a result for the case of $L$ near-field source localizations, the proposed algorithm replaces the $3-\mathrm{D}$ search required in the conventional $3-$ D MUSIC with a 2-D DOA search and $L$ $1-D$ range searches.

The notation that will be used throughout this article is as follows: $(\cdot)^{T},(\cdot)^{H},(\cdot)^{-1}, \operatorname{det}(\cdot)$, and $O(\cdot)$ denote transpose, Hermitian transpose, inverse, determinant, and big $\mathrm{O}$, respectively; $\operatorname{diag}\left(z_{1}, z_{2}\right)$ represents a diagonal matrix with diagonal entries $z_{1}$ and $z_{2}$.

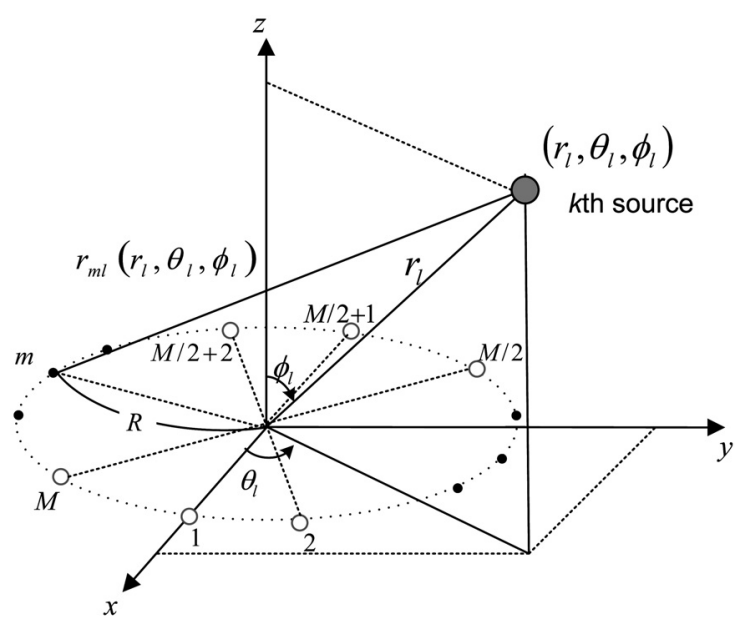

Fig. 1. Geometry of uniform circular array and source.

\section{Proposed algorithm}

The geometry of a UCA and source is shown in Fig. 1. A UCA consists of $M$ identical omnidirectional sensors, where $M$ is an even integer and the sensors are uniformly spaced along the circumference of a circle of radius $R$ in the $x y-$ plane. $L$ near-field uncorrelated narrowband sources are located at azimuth angles $\theta_{l} \in[0,2 \pi]$ measured counterclockwise from the $x$-axis, elevation angles $\phi_{l} \in[0,2 \pi]$ measured downward from the $z$-axis, and $r_{l}$ measured from the origin of the UCA.

The signal received at the $m$ th sensor can be modeled as

$$
x_{m}(n)=\sum_{l=1}^{L} s_{l}(n) e^{j \frac{2 \pi}{\lambda}\left\{r_{1}-r_{m}\left(r, \theta_{l}, \phi_{l}\right)\right\}}+w_{m}(n)
$$

for $m=1, \ldots, M, l=1, \ldots, L$, and $n=1, \ldots, N$, where $s_{l}(n)$ is the $l$ th source signal, $w_{m}(n)$ is the additive noise, $\lambda$ is the known wavelength, $N$ is the number of snapshots, and $r_{m l}\left(r_{l}, \theta_{l}, \phi_{l}\right)$ is the distance from the source located at $\left(r_{l}, \theta_{l}, \phi_{l}\right)$ to the $m$ th sensor, which is given by

$$
r_{m l}\left(r_{l}, \theta_{l}, \phi_{l}\right)=\sqrt{r_{l}^{2}+R^{2}-2 r_{l} R \xi_{m}\left(\theta_{l}, \phi_{l}\right)}
$$

where $\xi_{m}\left(\theta_{l}, \phi_{l}\right)=\cos \left((2 \pi / M) m-\theta_{l}\right) \sin \phi_{l}$. Using 
a second-order Taylor series expansion of $r_{m l}\left(r_{l}, \theta_{l}, \phi_{l}\right)$ for $m=1, \ldots . ., M$, it can be approximated as

$$
r_{m l}\left(r_{l}, \theta_{l}, \phi_{l}\right) \approx r_{l}\left\{1-\left(R / r_{l}\right) \xi_{m}\left(\theta_{l}, \phi_{l}\right)+\left(R^{2} /\left(2 r_{l}^{2}\right)\right)\left(1-\xi_{m}^{2}\left(\theta_{l}, \phi_{l}\right)\right)\right\}
$$

The second-order Taylor series approximation can be found in the literature on near-field source localization using UCA [3-4]. Using this approximation, the signal received in (1) can be reduced to

$$
\begin{aligned}
& x_{m}(n)=\sum_{l=1}^{L} s_{l}(n) e^{j \frac{2 \pi}{\lambda}\left\{R \xi_{m}\left(\theta_{l}, \phi_{l}\right)-\frac{R^{2}}{2 r_{l}}\left(1-\xi_{m}^{2}\left(\theta_{l}, \phi_{l}\right)\right)\right\}}+w_{m}(n), \\
& m=1, \ldots, M \text {. }
\end{aligned}
$$

The first term in the exponent of (4) is represented only by the 2-D DOA parameter of the source location, while the range parameter is related only to the second term in the exponent of (4). Therefore by applying Fresnel approximation, which corresponds to the secondorder Taylor expansion, the received signal model in the near field is decomposed into a range-independent $2-\mathrm{D}$ DOA parameters portion and a range-dependent $3-\mathrm{D}$ location parameters portion. The received signal vector $\mathbf{x}(n)=\left[x_{1}(n), \ldots, x_{M}(n)\right]^{T}$ can be written as

$$
\mathbf{x}(n)=\mathbf{A}(\mathbf{r}, \boldsymbol{\Theta}, \boldsymbol{\Phi}) \mathbf{s}(n)+\mathbf{w}(n)
$$

where $\mathbf{r}, \boldsymbol{\Theta}$, and $\boldsymbol{\Phi}$ are the $L$ dimensional source location vectors, $\mathbf{s}(n)=\left[s_{1}(n), \ldots, s_{L}(n)\right]^{T}$, $\mathbf{w}(n)=\left[n_{1}(n), \ldots, n_{M}(n)\right]^{T}$, and the direction matrix $\mathbf{A}(\mathbf{r}, \boldsymbol{\Theta}, \boldsymbol{\Phi})$ is defined as

$$
\begin{aligned}
\mathbf{A}(\mathbf{r}, \boldsymbol{\Theta}, \boldsymbol{\Phi}) & =\left[\mathbf{a}\left(r_{1}, \theta_{1}, \phi_{1}\right), \ldots, \mathbf{a}\left(r_{L}, \theta_{L}, \phi_{L}\right)\right] \\
\mathbf{a}\left(r_{l}, \theta_{l}, \phi_{l}\right) & =\left[a_{1}\left(r_{l}, \theta_{l}, \phi_{l}\right), \ldots, a_{M}\left(r_{l}, \theta_{l}, \phi_{l}\right)\right]^{T} \\
& {\left[\begin{array}{c}
j \frac{2 \pi}{\lambda}\left\{R \xi_{l}\left(\theta_{l}, \phi_{l}\right)-\frac{R^{2}}{2 r_{l}}\left(1-\xi_{l}^{2}\left(\theta_{l}, \phi_{l}\right)\right)\right\} \\
e^{j \frac{2 \pi}{\lambda}\left\{R \xi_{2}\left(\theta_{l}, \phi_{l}\right)-\frac{R^{2}}{2 r_{l}}\left(1-\xi_{2}^{2}\left(\theta_{l}, \phi_{l}\right)\right)\right\}} \\
\vdots \\
\left.e^{j \frac{2 \pi}{\lambda}\left\{R \xi_{M}\left(\theta_{l}, \phi_{l}\right)-\frac{R^{2}}{2 r_{l}}\left(1-\xi_{M}^{2}\left(\theta_{l}, \phi_{l}\right)\right)\right\}}\right]
\end{array}\right] }
\end{aligned}
$$

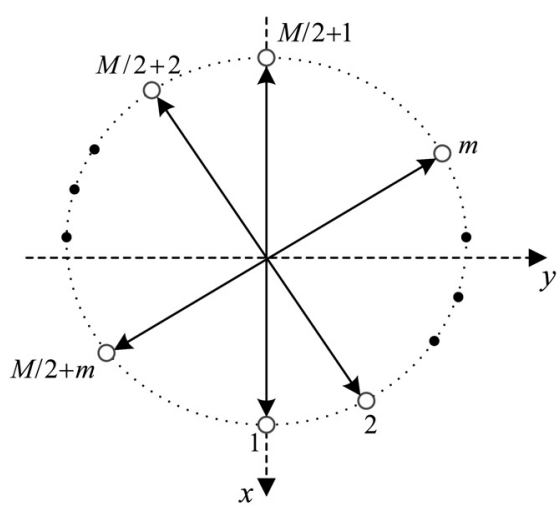

Fig. 2. The subarray structure for the RARE.

A RARE algorithm is applicable to sensor arrays consisting of several identically oriented and calibrated linear subarrays with the interelement spacing of each subarray being an integer multiple of some common baseline. Fig. 2 depicts the subarray structure for the RARE. As shown in Figure 2, a UCA can be composed of $M / 2$ identical linear subarrays consisting of the $m$ th and $M / 2+m$ th facing sensors.

Since the sensors are centrosymmetric, we have $\xi_{m}\left(\theta_{l}, \phi_{l}\right)=-\xi_{M / 2+m}\left(\theta_{l}, \phi_{l}\right) \quad[3-4,7]$. By reordering the sensors as

$$
\boldsymbol{\beta}_{R A R E}=[1, M / 2+1,2, M / 2+2, \ldots, M / 2, M],
$$

the steering vector $\mathbf{a}\left(r_{l}, \theta_{l}, \phi_{l}\right)$ in (7) can be rewritten as

$$
\begin{aligned}
\mathbf{a}\left(r_{l}, \theta_{l}, \phi_{l}\right) & =\left[a_{\beta_{1}}\left(r_{l}, \theta_{l}, \phi_{l}\right), \ldots, a_{\beta_{M}}\left(r_{l}, \theta_{l}, \phi_{l}\right)\right]^{T} \\
& =\left[\begin{array}{c}
e^{j \frac{2 \pi}{\lambda}\left\{R \xi_{l}\left(\theta_{l}, \phi_{l}\right)-\frac{R^{2}}{2 r_{l}}\left(1-\xi_{l}^{2}\left(\theta_{l}, \phi_{l}\right)\right)\right\}} \\
e^{j \frac{2 \pi}{\lambda}\left\{-R \xi_{l}\left(\theta_{l}, \phi_{l}\right)-\frac{R^{2}}{2 r_{l}}\left(1-\xi_{1}^{2}\left(\theta_{l}, \phi_{l}\right)\right)\right\}} \\
\vdots \\
e^{j \frac{2 \pi}{\lambda}\left\{R \xi_{M / 2}\left(\theta_{l}, \phi_{l}\right)-\frac{R^{2}}{2 r_{l}}\left(1-\xi_{M / 2}^{2}\left(\theta_{l}, \phi_{l}\right)\right)\right\}} \\
e^{j \frac{2 \pi}{\lambda}\left\{-R \xi_{M / 2}\left(\theta_{l}, \phi_{l}\right)-\frac{R^{2}}{2 r_{l}}\left(1-\xi_{M / 2}^{2}\left(\theta_{l}, \phi_{l}\right)\right)\right\}}
\end{array}\right] \\
& =\mathbf{C}\left(\theta_{l}, \phi_{l}\right) \mathbf{h}\left(r_{l}, \theta_{l}, \phi_{l}\right)
\end{aligned}
$$

where

$$
\mathbf{C}\left(\theta_{l}, \phi_{l}\right)=\left[\begin{array}{cccc}
\mathbf{c}_{1}\left(\theta_{l}, \phi_{l}\right) & \mathbf{0}_{2 \times 1} & \cdots & \mathbf{0}_{2 \times 1} \\
\mathbf{0}_{2 \times 1} & \mathbf{c}_{2}\left(\theta_{l}, \phi_{l}\right) & \cdots & \mathbf{0}_{2 \times 1} \\
\vdots & \vdots & \ddots & \vdots \\
\mathbf{0}_{2 \times 1} & \mathbf{0}_{2 \times 1} & \cdots & \mathbf{c}_{M / 2}\left(\theta_{l}, \phi_{l}\right)
\end{array}\right]
$$




$$
\begin{gathered}
\mathbf{c}_{i}\left(\theta_{l}, \phi_{l}\right)=\left[\begin{array}{c}
e^{j \frac{2 \pi}{\lambda}\left\{R \xi_{i}\left(\theta_{l}, \phi_{l}\right)\right\}} \\
e^{-j \frac{2 \pi}{\lambda}\left\{R \xi_{i}\left(\theta_{l}, \phi_{l}\right)\right\}}
\end{array}\right], i=1,2, \ldots, M / 2, \\
\mathbf{h}\left(r_{l}, \theta_{l}, \phi_{l}\right)=\left[e^{-j \frac{2 \pi R^{2}}{\lambda 2 r_{l}}\left(1-\xi_{l}^{2}\left(\theta_{l}, \phi_{l}\right)\right)}, e^{-j \frac{2 \pi R^{2}}{\lambda 2 r_{l}}\left(1-\xi_{2}^{2}\left(\theta_{l}, \phi_{l}\right)\right)}, \cdots, e^{-j \frac{2 \pi}{\lambda}\left(1-\xi_{\xi^{2} / 2}^{2}\left(\theta_{l}, \phi_{l}\right)\right)}\right]^{T},
\end{gathered}
$$

and $\beta_{m}$ for $m=1, \ldots, M$ denotes the $m$ th sensor index in the reordered sensor index vector $\boldsymbol{\beta}_{\text {RARE }}$ of (8), while $\mathbf{0}_{2 \times 1}$ is a $2 \times 1$ vector of zeros. Based on the reordering procedure, the steering vector $\mathbf{a}\left(r_{l}, \theta_{l}, \phi_{l}\right)$ is decomposed into a rangeindependent 2-D DOAs matrix $\mathbf{C}\left(\theta_{l}, \phi_{l}\right)$ and a range-dependent 3-D location vector $\mathbf{h}\left(r_{l}, \theta_{l}, \phi_{l}\right)$. An important observation following from (9) is that the range parameter $r$ is contained in $\mathbf{h}\left(r_{l}, \theta_{l}, \phi_{l}\right)$ only so that the matrix $\mathbf{C}\left(\theta_{l}, \phi_{l}\right)$ is independent of the range. Therefore to exploit this property for a range-independent 2-D DOA estimation we use the RARE algorithm.

The sample covariance matrix of the received signal reordered vector and its eigendecomposition are given as

$$
\mathbf{R}_{\mathbf{x}}=\frac{1}{N} \sum_{n=1}^{N} \mathbf{x}(n) \mathbf{x}(n)^{H}=\mathbf{E} \mathbf{\Lambda} \mathbf{E}^{H}+\mathbf{G} \boldsymbol{\Gamma} \mathbf{G}^{H}
$$

where $\mathbf{x}(n)$ is now the received signal reordered vector. The column vectors for $\mathbf{E}$ and $\mathbf{G}$ are the eigenvectors that span the signal and noise subspace of $\mathbf{R}_{\mathbf{x}}$, respectively, with the associated eigenvalues on the diagonals of $\boldsymbol{\Lambda}$ and $\boldsymbol{\Gamma}$.

Substituting the steering vector model in (9) to the MUSIC equation [6]

$$
\mathbf{a}^{H}\left(r_{l}, \theta_{l}, \phi_{l}\right) \mathbf{G G}^{H} \mathbf{a}\left(r_{l}, \theta_{l}, \phi_{l}\right)=0
$$

we have

$$
\mathbf{h}^{H}\left(r_{l}, \theta_{l}, \phi_{l}\right) \mathbf{B}\left(\theta_{l}, \phi_{l}\right) \mathbf{h}\left(r_{l}, \theta_{l}, \phi_{l}\right)=0
$$

where $\mathbf{B}\left(\theta_{l}, \phi_{l}\right)$ is a $(M / 2) \times(M / 2)$ matrix defined as

$$
\mathbf{B}\left(\theta_{l}, \phi_{l}\right)=\mathbf{C}^{H}\left(\theta_{l}, \phi_{l}\right) \mathbf{G G}^{H} \mathbf{C}\left(\theta_{l}, \phi_{l}\right) .
$$

because $\mathbf{h}^{H}\left(r_{l}, \theta_{l}, \phi_{l}\right) \neq 0$, (15) can be true only if $\mathbf{B}\left(\theta_{l}, \phi_{l}\right)$ drops rank (i.e., $\left.\operatorname{det}\left\{\mathbf{B}\left(\theta_{l}, \phi_{l}\right)\right\}=0\right)$. This property offers the basic rank-dropping criterion used in the RARE. It is worth noting that $\mathbf{B}\left(\theta_{l}, \phi_{l}\right)$ does not depend on any of the unknown rangerelevant vectors $\mathbf{h}^{H}\left(r_{l}, \theta_{l}, \phi_{l}\right)$. Therefore, the $2-\mathrm{D}$ DOAs can be estimated from the $L$ highest peak of the spectral RARE estimators in (17).

$$
\mathfrak{J}_{R A R E}(\theta, \phi)=\frac{1}{\operatorname{det}\{\mathbf{B}(\theta, \phi)\}}
$$

The peaks of the spectral RAREs in (17) indicate the estimated $2-\mathrm{D}$ DOAs $\left(\hat{\theta}_{l}, \hat{\phi}_{l}\right), l=1, \ldots, L$.

Once the 2-D DOAs are available, a range estimate can be independently estimated for each source. For example, for each given $\left(\hat{\theta}_{l}, \hat{\phi}_{l}\right)$ the range spectrum obtained by the $1-\mathrm{D}$ MUSIC yields

$$
\mathfrak{J}_{\text {MUSIC }}^{(l)}(r)=\left(\mathbf{a}^{H}\left(r, \hat{\theta}_{l}, \hat{\phi}_{l}\right) \mathbf{G} \mathbf{G}^{H} \mathbf{a}\left(r, \hat{\theta}_{l}, \hat{\phi}_{l}\right)\right)^{-1}, l=1, \ldots, L
$$

and the $l$ th range estimate can be determined from the global maxima using $\hat{r}_{l}=\arg \max _{r \in \mathbf{r}_{F}}\left[\mathfrak{I}_{M U S I C}^{(l)}(r)\right]$, where $\mathbf{r}_{F}$ is the Fresnel region.

The proposed algorithm replaces the $3-\mathrm{D}$ search required in the conventional 3-D MUSIC with a 2-D DOA search and $L$ 1-D range searches. The computational complexity of a 3-D MUSIC can be expressed by

$$
C_{3 D_{-} M U S I C}=\mathrm{O}\left(M^{2} N+M^{3}\right)+\mathrm{O}\left((M+2)(M-L) N_{\theta} N_{\phi} N_{r}\right) .
$$

The computational complexity of the proposed algorithm from (17) can be represented by

$$
\begin{aligned}
& C_{R A R E+1 D_{-} M U S I C}=\mathrm{O}\left(M^{2} N+M^{3}\right)+ \\
& \mathrm{O}\left(M^{2}\left(\frac{1}{8} M+\frac{5}{4}(M-L)\right) N_{\theta} N_{\phi}+L(M+2)(M-L) N_{r}\right),
\end{aligned}
$$


where $N_{\theta}, N_{\phi}$ and $N_{r}$ denote the number of grids in the $\theta$ space, $\phi$ space and $r$ space, respectively. In (19) and (20) the first term comes from the computation of a sample covariance matrix and its eigendecomposition, while the second term relates to the parameter search. Thus for the typical case of $N_{\theta}, N_{\phi}$ and $N_{r}>>M>L$, the computational burden for evaluating a sample covariance matrix and eigendecomposing the resultant matrix is inefficient. Therefore the computational complexity of these algorithms is related to the parameter search. The ratio of the computational complexity of the parameter searches employing the proposed algorithm using the RARE to that of using the 3-D MUSIC is approximately $\mathrm{O}\left(M / N_{r}\right)$ demonstrating the computational attractiveness of the proposed algorithm.

\section{Simulation}

This section investigates and validates the performance of the proposed estimator and compares the results with the benchmark estimator for the 3-D MUSIC. We consider a UCA consisting of $M=12$ with $R=\lambda / 2$, which is designed to avoid phase ambiguity, and two equi-power sources impinging on the array from $\left(r_{1}, \theta_{1}, \phi_{1}\right)=\left(2.5 R,-30^{\circ}, 60^{\circ}\right)$ and $\left(r_{2}, \theta_{2}, \phi_{2}\right)=$ $\left(3 R, 55^{\circ}, 45^{\circ}\right)$. Two hundred Monte Carlo runs with $N=256$ snapshots for each trial were performed to obtain the root-mean-squared errors (RMSEs) defined as $R M S E=\sqrt{\frac{1}{L M} \sum_{l=1}^{L} \sum_{m=1}^{M}(\text { estimated value }- \text { actual value })^{2}}$ for the 3-D location parameter. Note that the RMSE of the range estimate was normalized by the array radius $R$. A search grid with a step size of $(\Delta r, \Delta \theta, \Delta \phi)=\left(0.1 R, 0.1^{\circ}, 0.1^{\circ}\right)$ was applied to the proposed estimator and the $3-\mathrm{D}$ MUSIC.

Figs. 3 to 5 show that the estimation performance for the proposed estimator was sufficiently close to that of the 3-D MUSIC over a wide range of SNRs, despite the fact that the proposed

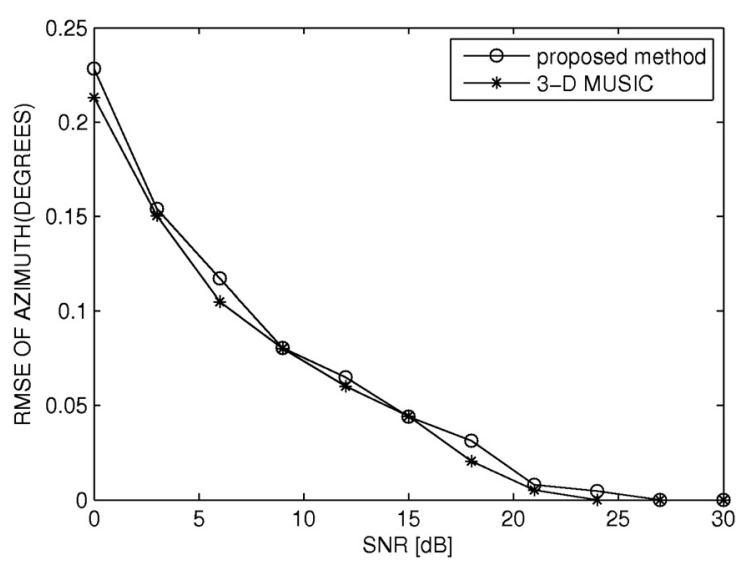

Fig. 3. RMSEs versus SNR of azimuth estimation.

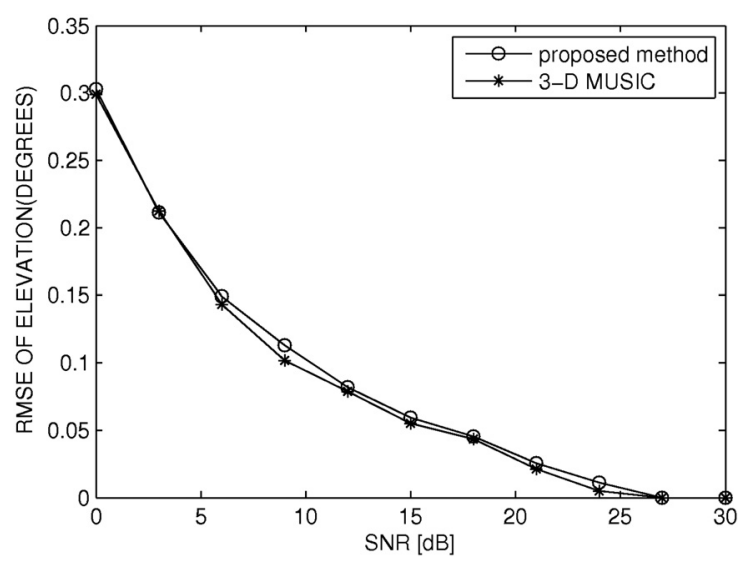

Fig. 4. RMSEs versus SNR of elevation estimation.

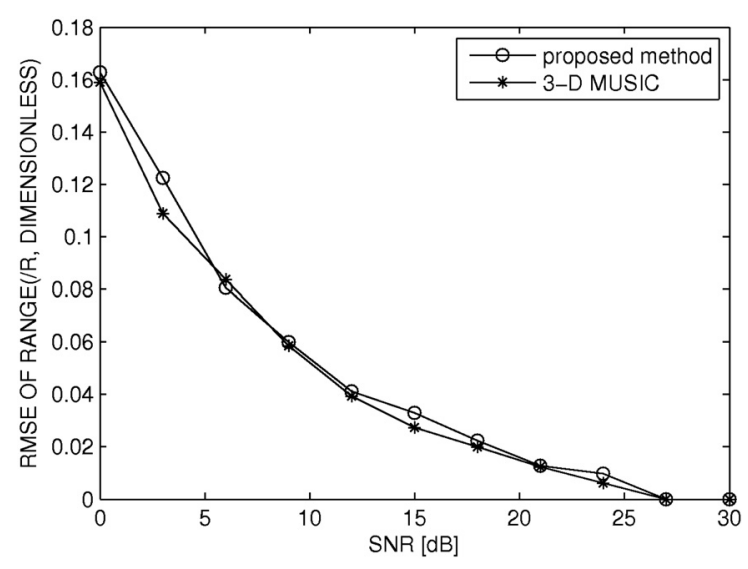

Fig. 5. RMSEs versus SNR of range estimation.

estimator does not demand an exhaustive grid search in contrast to the 3-D MUSIC.

The additional simulation compared the computational complexity of the proposed algorithm and 3-D MUSIC when using different numbers of sources, $L$. In this simulation, we assumed $N_{a z}=N_{e l}=N_{r a}=101$ search grids, where $N_{a z}$, 
Table 1. Elapsed CPU times required for parameter search.

\begin{tabular}{|c|c|c|c|c|}
\hline \multicolumn{2}{|c|}{ No. of sources } & $\mathrm{L}=1$ & $\mathrm{~L}=2$ & $\mathrm{~L}=3$ \\
\hline \multirow{2}{*}{ Proposed } & 2D-DOA & 0.1698 & 0.1688 & 0.1679 \\
\cline { 2 - 5 } & Range & 0.0017 & 0.0033 & 0.0048 \\
\hline \multicolumn{2}{|c|}{ 3-D Music } & 17.0421 & 17.0225 & 16.8667 \\
\hline \multicolumn{2}{|c|}{ Proposed/3-D MUSIC } & 0.0101 & 0.0101 & 0.0102 \\
\hline
\end{tabular}

$N_{e l}$, and $N_{r a}$ denote the number of grids in the azimuth space, elevation space, and range space, respectively. Table 1 lists elapsed CPU times used for the parameter search, which was computed by using the 'tic' and 'toc' run of MATLAB on a personal computer with a Intel i7 processor running at $2.8 \mathrm{GHz}$. The unit of the elapsed CPU time is second. The ratio of the elapsed CPU time of the proposed algorithm to that of 3-D MUSIC shows a slight increase as the number of sources increases. Yet, overall ratios are much smaller than ' 1 '.

\section{Discussion}

Our proposed algorithm replaces the $3-\mathrm{D}$ search required in the conventional 3-D MUSIC benchmark estimator with a 2-D DOA search and $\mathrm{L} 1-\mathrm{D}$ range searches. Using the RARE concept, our proposed algorithm develops a decoupled estimator, thereby providing a computationally efficient algorithm. And, despite its low computational complexity, the proposed algorithm can provide an estimation performance almost comparable to that of the conventional 3-D MUSIC benchmark estimator. Of course, the reference papers in [3-4] provide computationally efficient estimator. For example, Lee et al. [3] proposed a path-following algorithm allowing a 3-D location search to be replaced with a $2-D$ search for a far-field 2-D DOA estimation and 1-D search. However, under far-field assumption, a 2-D spectrum function of this algorithm for 2-D DOA has poor performance to distinguish multiple closely spaced sources. Furthermore, it requires path calculation procedure. Meanwhile, Bae et al. [4] developed a decoupled estimator for the 2-D DOA and range based on the centro-symmetry of the UCA and non-circularity of the source, which allows a computationally efficient, search-free near-field 3-D location estimator. However, its application is limited by the specific case of only a single noncircular source and the geometry of UCA with a center sensor. So, the reference paper in [4] cannot be directly compared to our algorithm because of the limited characteristics of source, the source number and the different array geometry. Bae et al. [4] considered the UCA with a center sensor and a single non-circular source. On the other hand, we considered multiple sources (including circular source and non-circular source) and the UCA without a center sensor. And, the radius of UCA is usually designed such that adjacent sensors are separated by less or equal to a half wavelength. However, in our proposed algorithm using rank reduction concept, the radius of UCA is designed by phase shift of two facing sensors. From (11), the phase shift of two elements is $\frac{4 \pi}{\lambda} R \xi_{i}\left(\theta_{i}, \phi_{i}\right)$. Therefore, the radius $R \leq \lambda / 2$ is assumed to avoid phase ambiguity.

\section{Conclusion}

A novel algorithm was proposed for nearfield 3-D localization of multiple sources in a UCA. Using a RARE algorithm the proposed method developed a decoupled estimator for the 2-D DOA and range, thus providing a computationally efficient algorithm. Numerical simulations showed that the proposed method yields an estimation performance comparable to that of the 3-D MUSIC algorithm, which requires an expensive $3-\mathrm{D}$ search procedure. 


\section{Acknowledgment}

This work was supported by Defense Acquisition Program Administration and Agency for Defense Development under the contract UD090002DD.

\section{References}

1. A. Y. J. CHAN and j. LITVA, "MUSIC and maximum likelihood techniques on two-dimensional DOA estimation with uniform circular array," IEE Proc., Radar Sonar Navig., vol. 142, no. 3, pp. 105-114., Jun. 1995.

2. Y. Wu and H. C. So, "Simple and accurate two-dimensional angle estimation for a single source with uniform circular array," IEEE Antennas Wireless Propag. Lett., vol. 7, pp. 7880, 2008.

3. J. H. Lee, D. H. Park, G. T. Park, and K. K. Lee, "Algebraic path-following algorithm for localizing $3-D$ near-field sources in uniform circular array," Electron. Lett., vol. 39, no. 17, pp. 1283-1285, Aug. 2003.

4. E. H. Bae and K. K. Lee, "Closed-form 3-D localization for single source in uniform circular array with a center sensor," IEICE Trans. Commun., vol. E92-B, no. 3, pp. 1053-1056, Mar. 2009

5. M. Pesavento, A. B. Gershman, and K. M. Wong, "Direction finding in partly-calibrated sensor arrays composed of multiple subarrays," IEEE Trans. on Signal Proc., vol. 50, no. 9, pp. 2103-2115, Sep. 2002.

6. R. O. Schmidt, "Multiple emitter location and signal parameter estimation," IEEE Trans. Antennas Propag., vol. 34, no. 3, pp. 276-280, May 1986.

7. Z. Ye, L. Xiang, and X. Xu, "DOA estimation with circular array via spatial averaging algorithm," IEEE Antennas Wireless Propag. Lett., vol. 6, pp. 74-76, 2007.

\section{【Profile】}

-Tae-Jin Jung

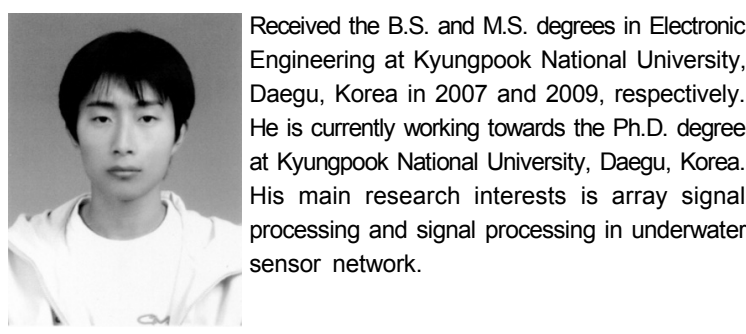

-Bum-Soo Kwon

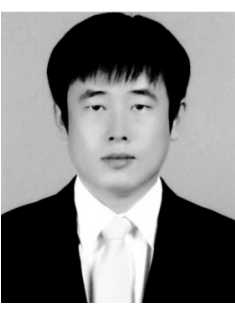

Received the B.S. and M.S. degrees in Electronic Engineering at Kyungpook National University, Daegu, Korea, in 2004 and 2006, respectively. $\mathrm{He}$ is currently working towards the Ph.D. degree at Kyungpook National University, Daegu, Korea. His main research interests is array and adaptive signal processing.

•Kyun Kyung Lee

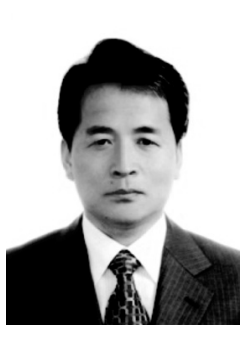

Received the B.S. degree at Sogang University, Seoul, Korea in 1977, his M.S. and Ph.D. degrees in Electronic Engineering from the University of Texas at Austin, USA, in 1984 and 1987 respectively. From March 1977 to February 1982 he was with Agency for Defense Development Korea. From March 1987 to February 1988 he was assistant professor in Oklahoma State University, USA. Since 1989, he joined the School of Electronic and Electrical Engineering, Kyungpook National University. Currently he is a professor in College of IT Engineering, Kyungpook National University, Korea and Director of Underwater Communication/Detection Research Center. His main research interests are Underwater Acoustic Signal Processing and Underwater Distributed Sensor Network. 